\title{
Improving self-care of patients with chronic disease using online personal health
}

\section{record}

\author{
Amol Wagholikar1 ${ }^{1}$, Maggie Fung², Colleen Nelson ${ }^{2}$
}

1. The Australian e-Health Research Centre, ICT Centre, CSIRO

2. The Australian Prostate Cancer Research Centre-Queensland, IHBI, QUT

\section{RESEARCH}

Please cite this paper as: Wagholikar A, Fung M, Nelson C. Improving self-care of patients with chronic disease using online personal health record. AMJ 2012, 5, 9, 517-521. http//dx.doi.org/10.4066/AMJ.2012.1358

Corresponding Author:

Dr Amol Wagholikar

The Australian e-Health Research Centre

(AEHRC) Level 5, UQ Health Sciences,

Building, RBWH, Herston, Queensland,

Australia, 4029

Email: Amol.wagholikar@csiro.au

\section{Abstract}

\section{Background}

Effective management of chronic diseases such as prostate cancer is important. Research suggests a tendency to use self-care treatment options such as over-the-counter (OTC) complementary medications among prostate cancer patients. The current trend in patient-driven recording of health data in an online Personal Health Record (PHR) presents an opportunity to develop new data-driven approaches for improving prostate cancer patient care. However, the ability of current online solutions to share patients' data for better decision support is limited. An informatics approach may improve online sharing of selfcare interventions among these patients. It can also provide better evidence to support decisions made during their selfmanaged care.

Aims

To identify requirements for an online system and describe a new case-based reasoning (CBR) method for improving self-care of advanced prostate cancer patients in an online PHR environment.

\section{Method}

A non-identifying online survey was conducted to understand self-care patterns among prostate cancer patients and to identify requirements for an online information system. The pilot study was carried out between August 2010 and December 2010. A case-base of 52 patients was developed.

\section{Results}

The data analysis showed self-care patterns among the prostate cancer patients. Selenium (55\%) was the common complementary supplement used by the patients. Paracetamol (about 45\%) was the commonly used OTC by the patients.

\section{Conclusion}

The results of this study specified requirements for an online case-based reasoning information system. The outcomes of this study are being incorporated in design of the proposed Artificial Intelligence (Al) driven patient journey browser system. A basic version of the proposed system is currently being considered for implementation.

\section{Key Words}

Advanced Prostate Cancer, Self-care, Patient Journey, PHR, Case-based reasoning

What this study adds:

1. This study reports self-care pattern among advanced prostate cancer patients by conducting a survey and specifies the requirements for an online system based upon survey findings and data analysis.

2. This study describes a case-based reasoning approach for improving accessible decision support information to the patients in an online PHR environment.

3. This study aims to make a contribution to the patientdriven online health data sharing solutions.

\section{Background}

Prostate cancer is one of the causes of male death in Australia. ${ }^{1}$ Prostate cancer was the most newly diagnosed cancer with 19,403 cases diagnosed in $2007 .^{2}$ Due to the chronic nature of the disease, it is difficult to understand the journey of patients especially those who are at an advanced stage. The journey of prostate cancer can be described using the various treatment points as events. ${ }^{3}$ Patients also uses several complementary supplements and OTC medications. It is important to understand self-care patterns of advanced prostate cancer patients with alternative therapies and OTC medications. It is also important to investigate the effect of collaboration among 
patients at various stages of the advanced prostate cancer journey. We conducted a survey for observing self-care pattern among advanced prostate cancer patients. We applied the survey findings to specify requirements for an online system and described a case-based reasoning approach to improve patient care in an online collaborative PHR environment. The research suggests a trend in online sharing of patient health data for better information to patients. ${ }^{4}$ Our research aims to leverage this trend to facilitate evidence-based decision support for advanced prostate cancer patients. Case-based reasoning (CBR) is a widely used approach for knowledge representation in $\mathrm{Al}$ driven applications. ${ }^{5-8}$ We have proposed a case-based reasoning approach to model the problem-solutionoutcome combination at various stages of the prostate cancer journey.

\section{Methods}

This research conducted a survey using a carefully designed questionnaire. The non-identifying questionnaire was designed to understand the care needs of the patients with advanced prostate cancer. The survey was designed using the categories such as demographics (e.g. age group), prostate cancer stage, care provider, treatment, medications, complementary or alternative therapy, dietary supplements, non-prescribed OTC medications and prescribed medications. The questionnaire was distributed to patients through prostate cancer support groups. The data collection period was from August 2010 to December 2010. The data was collected using an online version of the questionnaire as well as a paper version. A total of 52 patients participated in the pilot study. The data collected in this pilot study was used to answer the following research questions:

1. How many patients have ever used a complementary, alternative therapy or dietary supplements during or after conventional prostate cancer treatment?

2. What is the most common OTC drug used by these patients?

3. What are the co-morbidities in advanced prostate cancer patients?

\section{Results}

The survey findings showed that $59.62 \%$ of participants used a complementary, alternative therapy or dietary supplement during or after conventional prostate cancer treatment. Green tea, selenium (55\%) and tomatoes and lycopene were the most commonly used as a dietary intake; $49 \%$ of patients did not use any non-prescribed OTC medications. Patient who used any OTC medication commonly used paracetamol (45\%). High blood pressure was the most common co-morbidity among the patients.
The analysis of patient response in this study showed that $88 \%$ of patients were willing to participate in a suitable clinical trial. The analysis of age-related patient demographics shows that the percentage of patients aged greater than 60 years was $88 \%$. The comparison of the agerelated patient demographics with population-based findings specified in the report by the Australian Institute of Health and Welfare ${ }^{9}$ confirms that cancer incidence rate is higher in patients aged 60 years and above (mean age 68.4 for 2007). These survey results formed the foundation of our proposed technology based intervention to improve prostate cancer health outcomes. Our proposed system leverages trends in online health data sharing.

\section{Discussion}

\section{Mapping to the results of the survey}

The survey results provided an insight to various treatments undertaken by the advanced prostate cancer patients. The analysis of the survey results suggested a self-care pattern among advanced prostate cancer patients and showed a practice of self-reporting about the complementary and OTC medications. The survey results showed that the selfcare interventions undertaken by the patients are an integral part of understanding their journey. The patient reported data should be integrated with appropriate clinical treatment interventions undertaken and such integration will enable a holistic understanding of advanced prostate cancer patient's clinical journey.

\section{Rationale - arbitrary}

A patient journey is commonly represented as a work flow of patients from various health service providers. Our research into existing methods and tools shows that the existing systems mainly focus on work flow and data flow arising out of the work flow related to a particular health care setting. ${ }^{10,11}$ These systems are appropriate for enhancing business processes involved in providing care to the prostate cancer patients. However, clinical decisions can be made using a more granular level of patient data that integrates their clinical treatment information with selfadministered intervention. The information presented to the decision makers including carers as well as patients can be enhanced at a finer level of disease-specific details. In the context of self-management of the disease conditions, the patient data should be aggregated and presented to empower the patients. A self-management solution for advanced prostate cancer patients can provide them with a better understanding of their disease progression. There is a need for a new perspective towards modelling the patient journey. A patient's journey can be described in various health states. The health states can be represented by specific stages of advanced prostate cancer progression. 
The journey of men diagnosed with prostate cancer can be described in various health states (HS) such as localised prostate cancer, locally advanced prostate cancer and other advanced stages of the disease. Each health state can be characterised by specific clinical treatments, health outcomes achieved and any prostate cancer-specific side effects including mental health disorders such as anxiety and depression. Our research about existing online information resources for self-managed care of advanced prostate cancer has found that the information is mainly static and is of generic nature. The prostate cancer survivors need to process vast amount of generic information themselves and they frequently have to depend on their care providers for their personalised information. There is no clear evidence to suggest these existing methods and ways of distributing information to prostate cancer survivors are effective from health outcomes and health economics perspectives. Therefore, there is a need for information solutions that are more adaptable and personalised to empower prostate cancer survivors to take action for managing their own health especially during their rehabilitation. The proposed solution will add value to Prostate cancer survivors by providing personalised information to take actions for their self-managed care especially during their rehabilitation. Based on the findings of our survey, a research prototype was developed to describe sharing of complementary medication outcomes among prostate cancer patients. The purpose of the research prototype is to demonstrate efficient use of an online Personal Health Record (PHR) solution and selfreported data by the patients. It is assumed that the online PHR solution will allow patients to record data into their clinical record. The proposed system retrieves the clinical records from the various source systems. The unique contribution of our system is the collaborative access to self-care solutions through a CBR engine that retrieves the most similar problems-solution-outcome triple from the most similar patient record. The design of a case to be represented and stored in the CBR engine was given a lot of consideration. A case that models specific features or attributes of a patient's health state can be stored in different representational formats. ${ }^{12-15}$ The case features were chosen using the data items that are available or can be obtained. In the PHR environment, a patient can record data about their disease stage, complementary medication and health outcomes. The data structures for the features have to be available or be capable of being types that are available. $^{16}$ The features were chosen to facilitate an effective and efficient retrieval of cases stored in the case repository. The case-base was built using features: (i) current disease stage; (ii) complementary medication; (iii) outcome; (iv) outcome method. The case-base was developed using MySQL and the interface was developed using motion charts in Java Script. Figure 1 shows the interface of our research prototype.

Figure 1: Proposed solution prototype with a CBR engine

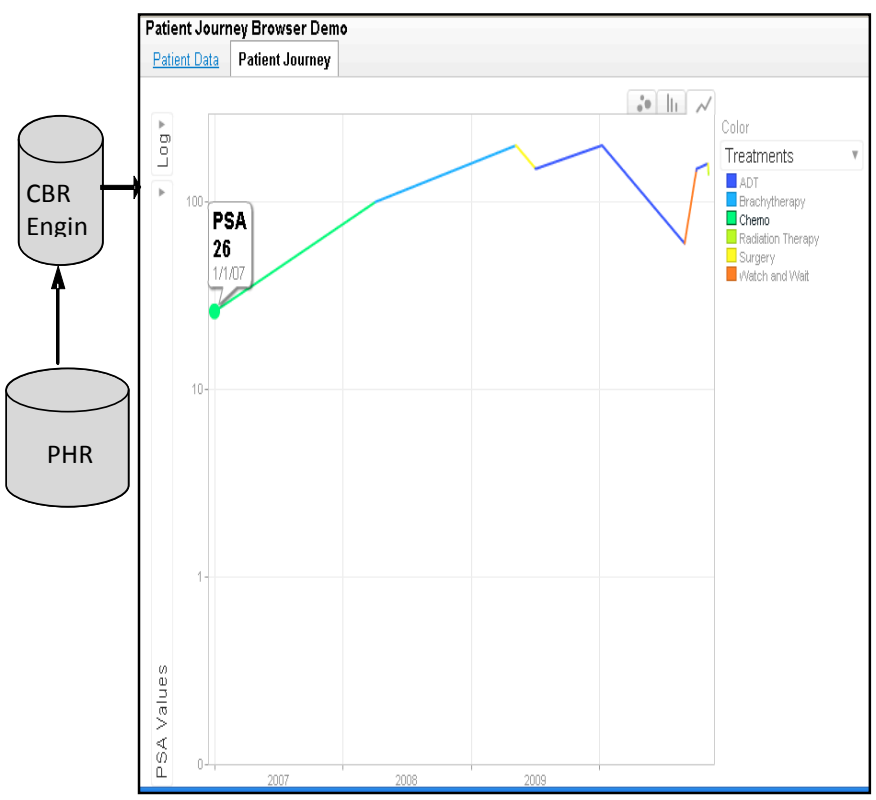

The computation of case similarity is a key aspect in CBR. The similarity is computed using Manhattan or city block distance measure. ${ }^{17}$ The similarity between two patient cases can be measured on an absolute scale. Manhattan or city-block distance measure is based on the absolute values of the case features. The similarity measure is derived from the method of computing the walking distance between two points in a city like New York's Manhattan district where each component is the number of blocks in northsouth and east-west directions. The formulation for the similarity between two patient cases is modelled as below:

$$
d_{i j}=\sum_{k=1}^{n}\left|x_{i k}-x_{j k}\right|
$$

Where $d_{i j}$ is the distance between data points in the casebase related to patient case. Each data point corresponds to the value of the features. The similarity computation determines the most similar case and the CBR engine retrieves it for sharing it with end-user. The end-user of our proposed system could be a patient searching for outcomes of complementary medication reported by patients on the same disease age. The cases were matched using the feature values of current disease stage and complementary medication. The disease stage and complementary medication are ordinal variables.

The features used in our approach are equally weighted for the simplicity of illustration of our prototype. The weights associated with the features can be determined by several 
methods and it is a separate research question. There can be various approaches for determining weights associated with the features in data aggregation problems especially in the context of case-based reasoning.

The described CBR approach facilitates the retrieval of the most similar patient case for sharing it with other patients at the similar disease stage. The visual data presentation is based on the changing values of prostate-specific antigen (PSA) levels over a given period in a line graph on logarithmic scale. The PSA test is a commonly used diagnostic measure for determining the disease progression of prostate cancer. The other key information such as current treatments, medications is displayed on the patient journey visual. The data visualisation assists patients in understanding their individual disease progression measured in PSA values. The data visualisation also assists patients in understanding the impact of treatments at specific disease stage. The care providers can also use the proposed visualisation in their clinical decision-making process.

The proposed approach could benefit patients in the selfmanagement of their disease conditions. The proposed system may provide evidence of effectiveness of OTC complementary medication used by patients at similar disease stages. This evidence will improve patient education. The patients may learn about effective interventions by knowing about similar patient journeys. The patients may also use the proposed system to undertaken preventative actions in consultation with their primary clinicians. The proposed solution can also be integrated with existing social networks as patients or users with chronic conditions will be able to share their specific journey with other users of their choice. The proposed system is a research prototype with the potential to generate new evidence of effectiveness of specific interventions at each health state. A full scale commercial grade development and evaluation will be undertaken in future. The proposed approach presents a new perspective of modelling journey of patients with chronic diseases using Al techniques and data driven methods.

\section{Limitations}

The scope of this research is limited to advanced prostate cancer patients. The data collection from a large number of patients was challenging due to limitations in reaching out to advanced prostate cancer patients. Many patients did not attend the patient support groups. The patients who participated in the survey represent the target population of advanced prostate cancer patients at a smaller scale. The scarcity of data about treatment patterns especially during advanced stages of prostate cancer is a limitation encountered during our research. The challenge in acquiring a statistically sufficient sample size of advanced prostate cancer can be addressed by building a national registry of advanced prostate cancer patient data for research purposes. We acknowledge that methods of determining confidence in disease stage estimation are yet to be explored. We propose to address this challenge in future through a large scale study of the proposed research. It is anticipated that the limitations specified in this paper can be addressed in a large scale study.

\section{Conclusion}

We showed that advanced prostate cancer patients use a complementary medication which suggests a pattern of selfcare among the patients. This study has described a CBRbased approach to improve self-care for advanced prostate cancer patients in an online PHR environment. We showed that a technology-based intervention can improve data access to advanced prostate cancer patients. A basic version of the proposed system is being considered for use at an advanced prostate cancer multidisciplinary team clinic at a tertiary hospital in Queensland.

\section{References}

1. Cancer Council Australia. Available from: www.cancer.org.au//aboutcancer/cancertypes/prostate cancer.htm. Accessed 24 January 2011.

2. Jemal A, Siegel R, Ward E, Hao Y, Xu J, Thun MJ. Cancer Statistics, 2009. CA Cancer J Clin. 2009 Jul-Aug; 59(4):225-49. Epub 2009 May 27.

3. Advanced prostate cancer: a guide for men and their families. Available from: www.andrologyaustralia.org/docs/Advanced_Prostate_ Cancer_Guide_2009.pdf. Accessed 24 January 2011.

4. Wicks P, Massagli M, Frost J, Brownstein C, Okun S, Vaughan $T$ et al. Sharing health data for better outcomes on PatientsLikeMe. J Med Internet Res. 2010 Jun 14; 12(2):e19.

5. Althoff KD. Case-based Reasoning, In Chang SK (editor), Handbook of Software Engineering \& Knowledge Engineering. New Jersey, USA. World Scientific; 2001.

6. Kitano $H$, Shimazu $H$. The experience sharing architecture: a case study in corporate-wide case-based software quality control. In: Leake DB (editor), Casebased Reasoning: Experiences, Lessons, and Future Directions. Menlo Park, CA. AAAI Press/The MIT Press; 1996.

7. Sun Z, Finnie G. Intelligent techniques in E-Commerce: A Case Based Reasoning Perspective. Berlin. PhysicaVerlag; 2003. 
8. Plaza E, Aamodt A. Case-based reasoning, Foundational Issues, Methodological Variations, and System Approaches. Artificial Intelligence Communications. 1994; 7(1): 39-59.

9. Australian Institute of health and Welfare, Cancer in Australia 2010: An overview. Available from: www.aihw.gov.au/publications/can/56/12138.pdf. Accessed: 24 January 2011.

10. McGregor C, Steadman A, Percival J, James A. A Method for Modeling Health Informatics Capacity in Patient Journeys Supported by Interprofessional Teams. In: Sprague, RH (editor) Proceedings of the 45th Hawaii International Conference on Systems Sciences, HICSS'45, ISBN: 978-0-7695-4525-7, Maui, Hawaii, January 4-7 2012. pp 2790-99.

11. Curry J, McGregor C. The current state of patient journey redesign in Australia. In Wise $\mathrm{M}, \mathrm{Grain} \mathrm{H}$, Chu S (editors) HIC 2005 and HINZ 2005 Proceedings. Brunswick East, Vic.: Health Informatics Society of Australia, 2005, ISBN: 0975101358, Melbourne, Australia, July 31-Aug 2, 2005, pp.323-331.

12. Pal S, Shiu S. Foundations of Soft Case-Based Reasoning. Hoboken, NJ. Wiley-Interscience; 2004.

13. Gebhardt F, Vo A, Grather W, Schmidt-BelzB. Reasoning with Complex Cases, International Series in Engineering and Computer Science, Vol. 393. Boston. Kluwer Academic Publishers; 1997.

14. Pal SK, Dillon TS, Yeung, DS (eds). Soft Computing in Case Based Reasoning. London. Springer-Verlag; 2000.

15. Bergmann R. Experience Management: Foundations, Development Methodology, and Internet-Based Applications. Berlin. Springer; 2002.

16. Cortés $U$, Núñez $H$, Sànchez-Marrè $M$, Comas J, Ignasi $R$, Poch, M. Feature Weighting Techniques for Prediction Tasks in Environmental Processes. ECAl Workshop on Binding Environmental Sciences and Artificial Intelligence (BESAl'2002), Lyon, France; 2002.

17. Plaza E, Esteva P, Garcia L, Godo R., de Mantaras LA. Logical approach to case-based reasoning using fuzzy similarity relations. International Journal of Information Science. 1996; 106: 105-122.

\section{ACKNOWLEDGEMENTS}

This research is a collaborative project between the Australian Prostate Cancer Research Centre-Queensland at Queensland University Technology (QUT) and the Australian e-Health Research Centre at Commonwealth Scientific and Industrial Research Organisation (CSIRO). The researchers sincerely thank the collaborators, the Prostate Cancer Support Groups and Prostate Cancer Foundation of Australia (PCFA) for their valuable cooperation.

\section{PEER REVIEW}

Not commissioned. Externally peer reviewed

\section{CONFLICTS OF INTEREST}

None

\section{FUNDING}

The research was funded by the collaborative research project grant between the AEHRC and QUT.

\section{ETHICS COMMITTEE APPROVAL}

Ethics approval for the de-identified data collection from the patients was granted by the Human Research Ethics Committee, Queensland University of Technology (QUT). This study was granted an ethics approval by Queensland Government's Human Research Ethics Committee (HREC); Ethics Approval No: HREC/11/QPAH/317. 